\title{
Radio Emission from SN 1987A
}

\author{
By L. STAVELEY-SMITH ${ }^{1}$, R. N. MANCHESTER ${ }^{1}$, \\ A. K. TZIOUMIS $S^{1}$, J. E. REY NOLD $\mathbf{S}^{1}$, \\ AND D. S. BRIG G S \\ ${ }^{1}$ Australia Telescope National Facility, CSIRO, PO Box 76, Epping, NSW 2121, Australia \\ ${ }^{2}$ National Radio Astronomy Observatory, PO Box 0, Socorro, NM 87801, USA
}

We review the first six years of radio observations of Supernova 1987A. The evolution can be divided into two phases: the initial radio outburst which lasted a few weeks, and the period from mid-1990 to the present, during which the radio emission has steadily increased. Both phases can be explained by a small fraction (0.1-0.5\%) of the post-shock thermal energy being converted to energy in relativistic particles and magnetic fields, which give rise to synchrotron radiation. The optical depths, densities and density profiles for the pre-shocked circumstellar material are somewhat different for the two phases, but consistent with models of the density structure of the material within the circumstellar ring. New high-resolution radio observations show that the SN shock front is already at about three-quarters of the radius of the circumstellar ring, and that there exists a bright equatorial component of emission aligned with this ring which is probably due to a polar density gradient in the 'hourglass' structure.

\section{Introduction}

Radio studies of supernovae began with the detection of SN 1970G in M101 (Gottesman et al. 1972; Allen et al. 1976), though it wasn't for another decade that detailed radio light curves were available for a statistically useful sample of supernovae. Mainly through the work of Weiler, Sramek and collaborators (this volume) at the Very Large Array, there are now over a dozen well-studied examples of radio supernovae (RSN). These observations have been useful mainly in exploring the distribution of circumstellar material and therefore the progenitor mass-loss history (e.g. Weiler et al. 1989). However, other applications such as the local distance scale from geometric expansion (Bartel et al. 1985) and probing the interstellar medium through spectral line absorption studies (Ryder et al. 1993) are possible. Supernova 1987A has offered us a chance of exploring all of these aspects in detail, and also of following the evolution through the RSN phase and into the much later supernova remnant (SNR) phase, giving us for the first time a direct view of this transition. Additionally, the surprising fact that the progenitor was a blue supergiant, with a much lower density wind, allows us to study an example of a supernova that would not have been detected in the radio outside the Local Group of galaxies.

\section{The Prompt Outburst}

Radio emission from SN $1987 \mathrm{~A}$ peaked at $140 \mathrm{mJy}$ at $843 \mathrm{MHz}$ about three days after the explosion (Fig. 1 and Turtle et al. 1987). Emission at higher frequencies peaked slightly earlier, probably as a result of lower thermal absorption. If modelled as the interaction of the shock with an $r^{-2}$ stellar wind, Chevalier and Fransson (1987) estimate a mass-loss rate $\dot{M}=8.8 \times 10^{-6}\left(V_{w} / 550 \mathrm{~km} \mathrm{~s}^{-1}\right)\left(u / 26,000 \mathrm{~km} \mathrm{~s}^{-1}\right)^{3 / 2}\left(T / 10^{5} \mathrm{~K}\right)^{0.75} \mathrm{M}_{\odot}$ $\mathrm{yr}^{-1}$, and a non-thermal energy density $\sim 0.1 \%$ of the thermal energy density. Because of clumping and contributory processes such as internal free-free absorption, which can 


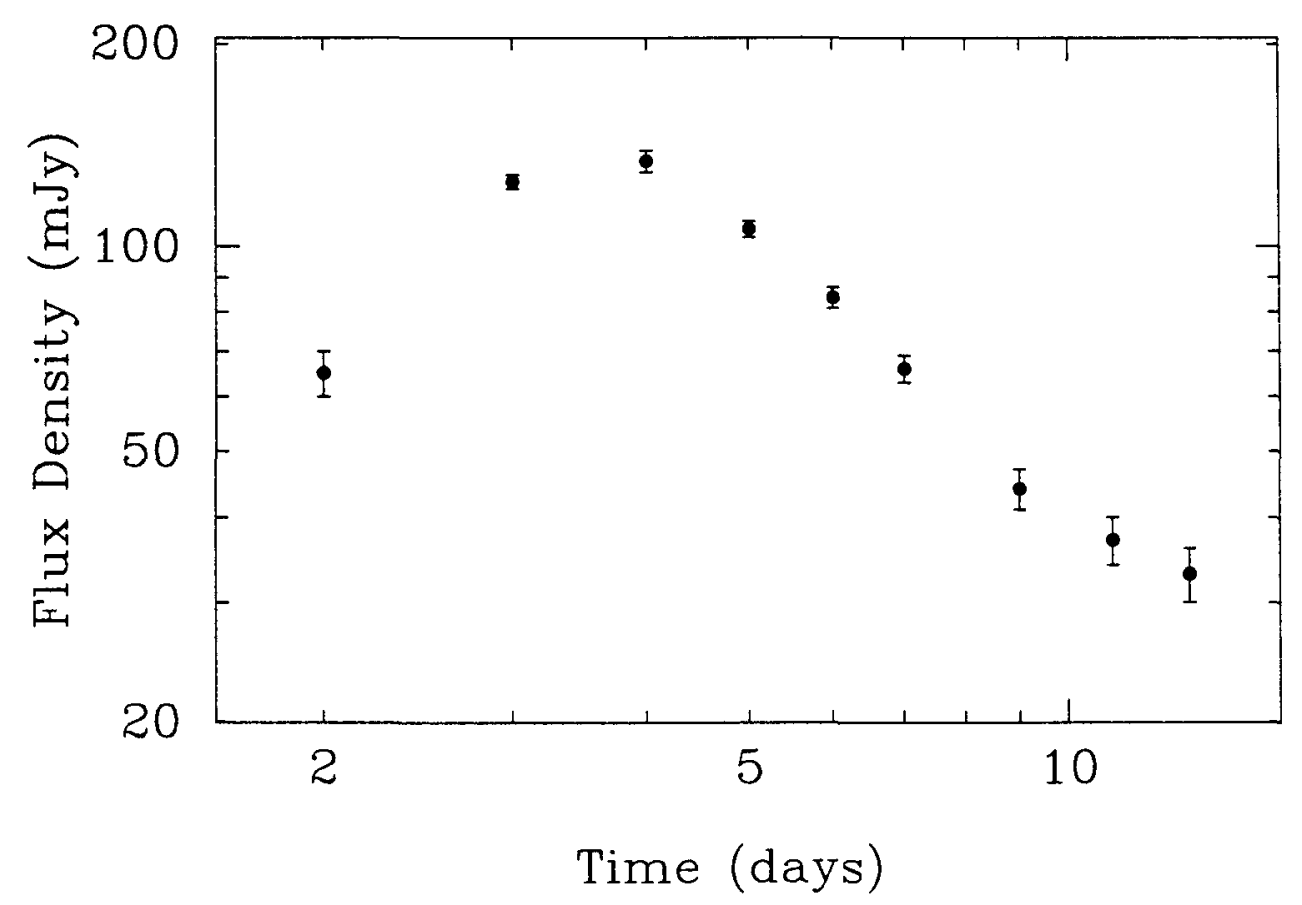

FIgURE 1. $843 \mathrm{MHz}$ radio emission for SN 1987A for two weeks after outburst (from Turtle et al. 1987).

arise from Compton cooling of the ejecta at early times (Chevalier \& Fransson 1987) and synchrotron self-absorption (Storey \& Manchester 1987; Kirk \& Wassmann 1992), this mass-loss rate is an upper-limit. After a few months, no radio emission was detectable.

Very long baseline interferometry observations were attempted very early on, but no emission was detected, implying that the radio photosphere was already resolved out (Jauncey et al. 1988). An initial shock velocity $>19,000 \mathrm{~km} \mathrm{~s}^{-1}$ was implied, which is consistent with early optical spectroscopy (Hanuschik \& Dachs 1987).

\section{Interaction with the Bubble}

Radio emission was again detected from SN 1987A in 1990 July by the Molonglo Observatory Synthesis Telescope (MOST) at $843 \mathrm{MHz}$ and the Australia Telescope Compact Array (ATCA) at 1.4 and $5 \mathrm{GHz}$ (Turtle et al. 1990). Flux densities at these frequencies in 1990 mid-August were $4.5,2.0$ and $0.7 \mathrm{mJy}$, respectively. Since then, the flux density has increased steadily at all frequencies (Staveley-Smith et al. 1992) to its present level of $\sim 12 \mathrm{mJy}$ at $5 \mathrm{GHz}$. ATCA observations at $8.6 \mathrm{GHz}$ show that the radio remnant is $\sim 0^{\prime \prime} .5$ from the optical position reported for SN 1987A by White and Malin (1987), and others. Although higher than expected, it is not outside the errors present in the Perth 70 catalogue. Resolution of the slight position discrepancy awaits data from the Hipparcos satellite. The spectrum of the radio emission in this phase is nonthermal $\left(S_{\nu} \propto \nu^{-1.0}\right)$, with no sign of a free-free turnover, in contrast to the prompt 


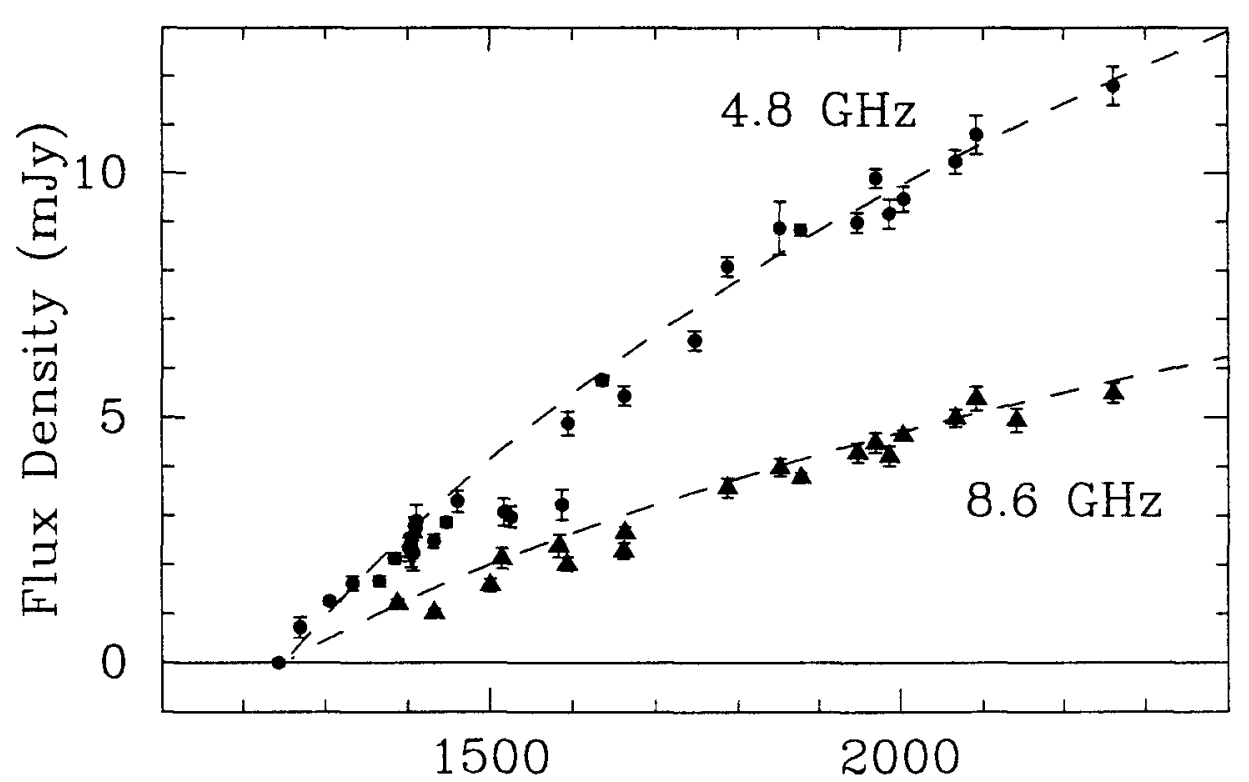

Time (days)

Figure 2. Radio emission from SN $1987 \mathrm{~A}$ between days 1200 and 2300 at $4.8 \mathrm{GHz}$ (circles) and $8.6 \mathrm{GHz}$ (triangles). Observations taken with the Australia Telescope Compact Array. The fits are for the $\gamma=3, s=0, m=0.65$ circumstellar interaction model described in the text.

outburst and the rising portions of the light curve of all previously observed RSNs. The $10 \mathrm{MHz}-10 \mathrm{GHz}$ radio luminosity of $1.1 \times 10^{33} \mathrm{erg} \mathrm{s}^{-1}$ (1993.3) is small compared with other SNRs and RSNs $\left(\sim 10^{-2} \times\right.$ Cas A and $\sim 10^{-5} \times$ SN 1986J at its peak, Rupen et al. 1987). The minimum energy required in cosmic rays and magnetic fields to produce the synchrotron emission is $1.8 \times 10^{47} \phi^{3 / 7}$ erg (Pacholczyk 1970), assuming that $1 \%$ of the cosmic ray energy is in electrons, and a radiating volume of $0.017 \phi$ $\mathrm{pc}^{3}$, where $\phi$ is the volume filling factor. The magnetic field at minimum energy is $3.4(\phi / 0.2) \mathrm{mG}$, and the ratio of the minimum energy to the post-shock thermal energy is $5 \times 10^{-3}(\phi / 0.2)^{-4 / 7}\left(n_{e} / 6 \mathrm{~cm}^{-3}\right)^{-1}\left(u / 35,000 \mathrm{~km} \mathrm{~s}^{-1}\right)^{-2}$, where $n_{e}$ is the unshocked circumstellar thermal electron density and $u$ is the shock velocity.

Fits to the observed flux density increase have been made by Ball and Kirk (1992). Using a diffusive shock acceleration model with a constant acceleration of relativistic electrons, they were able to produce acceptable fits to the early light curves at $843 \mathrm{MHz}$ and $5 \mathrm{GHz}$. Chevalier (1992) produced a model in which the radio emission was generated by the interaction of the supernova shock wave with the density jump associated with the termination radius of the blue supergiant progenitor wind. The termination radius was calculated to be $\sim 3 \times 10^{17} \mathrm{~cm}$ or about half the radius of the circumstellar ring, which fitted with the measured radius of the radio source. However, this acceleration mechanism is limited in its duration by the acceleration timescale and the differential light travel time. Chevalier (1992) estimated a rise time of one year, whereas the light curve has not yet flattened off, even after three years (see Fig. 2). 


\section{Models}

Given the steady increase of the radio emission in the second phase, it is more likely that there is continuous particle acceleration and magnetic field compression. The ultimate source of the energy required for this is the expanding shock wave as it interacts with the surrounding medium. We assume a circumstellar density profile $\rho_{c} \propto r^{-s}$, and an expansion law for the supernova shock front $r \propto t^{m}$. At a given radius the post-shock thermal energy density $\left(\rho_{c} u^{2}\right)$ is therefore

$$
E_{p s}(r) \propto \rho_{\circ} m^{2} u_{o}^{2} t^{2 m-2-m s} .
$$

The volume synchrotron emissivity, in the optically thin regime, is

$$
j_{\nu} \propto K B^{\frac{1+\gamma}{2}} \nu^{-\left(\frac{\gamma 1}{2}\right)},
$$

(Ginzburg \& Syrovatskii 1965) where $B$ is the magnetic field and $\gamma$ is the index of the differential electron energy spectrum $N(E) d E \propto K E^{-\gamma} d E$. Following Chevalier (1982a), if we assume that a constant fraction of $E_{p s}$ goes into relativistic electron energy density $(\propto K)$ and magnetic field energy density $\left(\propto B^{2}\right)$, then the volume synchrotron emissivity becomes

$$
j_{\nu} \propto\left(\rho_{\circ} m^{2} u_{\circ}^{2}\right)^{\frac{5+\gamma}{4}} t^{(2 m-2-m s) \frac{5+\gamma}{4}} \nu^{-\left(\frac{\gamma 1}{2}\right)} .
$$

The monochromatic synchrotron luminosity $L_{\nu}$ depends on acceleration time scales, loss processes and differential light travel time, but taking the approximation $L_{\nu}=\frac{4}{3} \pi \phi j_{\nu} r^{3}$, we have:

$$
L_{\nu} \propto \phi\left(\rho_{\circ} m^{2} u_{\circ}^{2}\right)^{\frac{5+\gamma}{4}} t^{(22 m-10-m s(\gamma+5)+2 \gamma(m-1)) / 4} \nu^{-\left(\frac{\gamma 1}{2}\right)} .
$$

For $s=2$ (the time invariant stellar wind solution), this reduces to a time-dependence $L_{\nu} \propto t^{-(5+\gamma-6 m) / 2}$, which is identical to the solution given in Chevalier (1982a). When combined with thermal absorption, this fits the observations of the prompt outburst (as above), and works reasonably successfully for other RSNs (Weiler et al. 1989). For SN $1987 \mathrm{~A}$, with a radio spectral index $\alpha=1.0\left(L_{\nu} \propto \nu^{-\alpha}\right)$, and $\alpha=(\gamma-1) / 2$, we require an accelerating shock front $(m>4 / 3)$ to achieve a radio flux density that increases with time in the optically thin regime. This is unphysical.

However, the $s=2$ solution is not appropriate at the present shock radius. Models of stellar evolution and observations of the photoionized medium around SN 1987A imply that the blue supergiant phase only lasted for $2 \times 10^{4}$ yr (Crotts \& Heathcote 1991). Before this, the star was a red supergiant with a much denser, slower wind. The interaction of fast winds with slow-moving, or stationary, media has been described elsewhere (Dyson \& de Vries 1972; Castor et al. 1975; Falle 1975). A number of distinct physical regions are formed (Fig. 3 and Wang \& Mazzali 1992). Between the free winds from the blue and red supergiant phases, lies a shocked wind region of approximately constant density. In the case of SN 1987 A, this lies between the circumstellar ring and the termination radius mentioned earlier. Can the synchrotron luminosity increase in the region? Take the simplifying case with $s=0$ and assume that the fast wind termination radius is small enough that the solution remains self-similar. Then we have:

$$
L_{\nu} \propto \phi\left(\rho_{\circ} m^{2} u_{\circ}^{2}\right)^{\frac{5+\gamma}{4}} t^{(22 m-10+2 \gamma(m-1)) / 4} \nu^{-\left(\frac{\gamma 1}{2}-\right)} .
$$

With $\gamma=3$ (as above), $L_{\nu}$ will increase with time for $m>4 / 7$. The expansion index $m$ is related to the density profile of the stellar ejecta $\rho_{*} \propto r^{-n}$ by $m=(n-3) /(n-s)$ (Chevalier 1982b), which corresponds to $n>7$. To apply this to SN $1987 \mathrm{~A}$, we take the above approximation and assume that the radio emission only turns on at time $t_{\mathrm{o}}$ after 


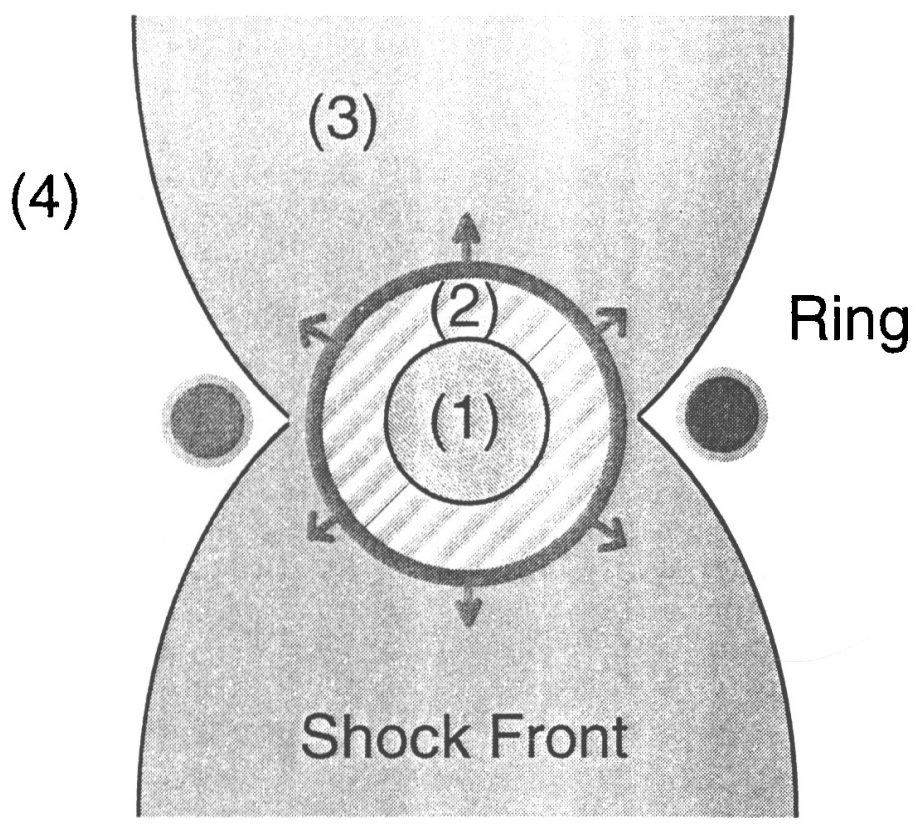

FIGURE 3. The relationship of the SN 1987A shock front to the circumstellar environment. Regions (1) and (2) have been overtaken by the SN shock. Region (1) was occupied by the free wind from the blue supergiant. Region (2) was occupied by the blue supergiant wind, heated up by interaction with the red supergiant wind; region (3) is the same as (2), but has yet to be hit by the SN shock. Region (4) is the free red supergiant wind which the SN shock has yet to reach.

the explosion:

$$
\phi(r)=1-\left(\frac{t}{t_{0}}\right)^{-3 m}, \quad t \geq t_{\circ}
$$

and $\phi(r)=0$ otherwise. For times $t \geq t_{0}$, we obtain

$$
L_{\nu} \propto \rho_{\circ}^{2}\left(m u_{\circ}\right)^{4}\left[1-\left(\frac{t}{t_{\circ}}\right)^{-3 m}\right] t^{7 m-4} \nu^{-\left(\frac{\gamma^{1}}{2}\right)} .
$$

A reasonable fit to the data in Fig. 2 is obtained with $t_{0}=1250$ days and $m=0.65$, corresponding to $n=8.6$ which is similar to the values obtained by Arnett (1988) from fits to the optical light curve at early stages. Between days 1400 and 1700 the fit in Fig. 2 is not good. The constant energy injection model of Ball \& Kirk (1992) gives somewhat better fits up to day 1800 by assuming a two-component model and a finite electron acceleration time scale. However, their fit deviates beyond day 1800 . Clearly, future work will require a better understanding of electron acceleration and shock deceleration processes, the incorporation of differential light travel time and modifications required by recent high-resolution imaging which imply deviations from spherical symmetry.

\section{High-Resolution Imaging}

The present size of the remnant (radius $\sim 0^{\prime \prime} .6$ ) unfortunately lies between the capabilities of $6 \mathrm{~km}$ ATCA (highest diffraction limit $0^{\prime \prime} .85$ at $9 \mathrm{GHz}$ ) and the Australian Long Baseline Array (lowest resolution $\sim 0^{\prime \prime} .1$ at $1.4 \mathrm{GHz}$, for an imaging observation). However, the increasing source strength of SN 1987 A and recent improvements in ATCA 


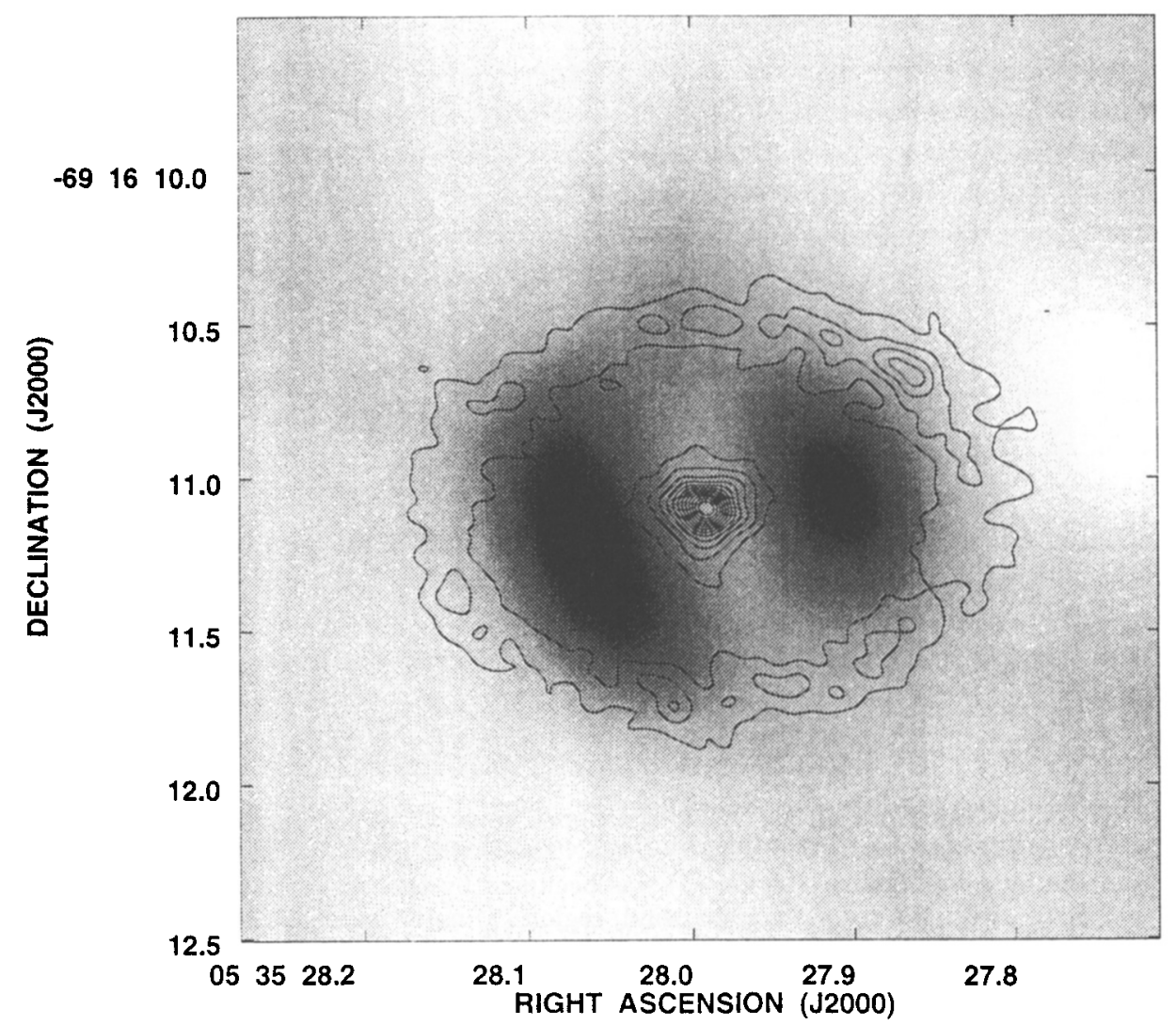

FIgure 4. Super-resolved observation of SN $1987 \mathrm{~A}$ at $8.8 \mathrm{GHz}$ (ATCA). Data from 1992 October and 1993 January were used to make the image (Staveley-Smith et al. 1993). The resolution is $\sim 0^{\prime \prime} .5$. A contour plot of a Hubble Space Telescope [OIII] observation (Jakobsen et al. 1991) has been overlaid (assuming that the SN lies at the centre of the radio remnant).

receiver sensitivity have allowed the use of 'super-resolution' techniques which can improve on the canonical diffraction limit. Using very long integration times $(3 \times 12 \mathrm{hr})$ and high bandwidths $(2 \times 128 \mathrm{MHz})$ at 8.64 and $8.90 \mathrm{GHz}$ during observations between 1992 October and 1993 January, Staveley-Smith et al. (1993) were able to employ maximum entropy deconvolution procedures to achieve an angular resolution of $0^{\prime \prime} .5$, or $3.6 \times 10^{17}$ $\mathrm{cm}$, a factor of 1.7 below the diffraction limit. This is sufficient to reveal that the remnant has a limb-brightened structure with two prominent hotspots aligned along the equatorial plane defined by the slightly more distant circumstellar ring (Fig. 4). The mean inferred radius of the remnant is $3.2 \times 10^{17} \mathrm{~cm}$, although model fitting suggests that the true outer radius is probably at $\sim 4.7 \times 10^{17} \mathrm{~cm}$, which is $76 \%$ of the radius of the circumstellar ring.

The alignment of the radio hotspots with the circumstellar ring suggests that constant density approximation for region (3) needs modifying. The implication is that the shock wave is now passing through a denser medium in the equatorial plane. This may be due to a general polar density gradient, or from the shock wave passing through high density clouds sheared from the interface between regions (3) and (4).

The mean expansion rate (radius/time) inferred from visibility modelling is $34600 \pm$ $1200 \mathrm{~km} \mathrm{~s}^{-1}$ at day 1500 , but only $25900 \pm 400 \mathrm{~km} \mathrm{~s}^{-1}$ at day 2060 . This large apparent deceleration may be due partly to an increase in density towards the circumstellar ring, 
which is reflected in the enhanced radio emission in this region. More likely, however, is that the remnant is changing in form and that the visibility modelling procedure has been accordingly biased. For example, it is likely that the volume filling factor $\phi$ has a time-dependence as discussed above. A true measurement of the deceleration awaits a second-epoch high-resolution observation. Such an observation will be the only direct way to predict the date of onset of the interaction between the shock front and the circumstellar ring.

\section{REFERENCES}

Allen, R. J., Goss, W. M., Ekers, R. D. \& de Bruyn, A. G. (1976). Astr. Astrophys., 48, 253-261. Arnett, W. D. (1988). ApJ, 331, 377-387.

Ball, L. \& Kirk, J. G. (1992). ApJ, 396, L39-L42.

Bartel, N., Rogers, A. E. E., Shapiro, I. I., Gorenstein, M. V., Gwinn, C. R., Marcaide, J. M. \& Weiler, K. W. (1985). Nature, 318, 25-30.

Castor, J., McCray, R. \& Weaver, R. (1975). ApJ, 200, L107-L110.

Chevalier, R. A. (1982a). ApJ, 259, 302-310.

Chevalier, R. A. (1982b). ApJ, 258, 790-797.

Chevalier, R. A. (1992). Nature, 355, 617-618.

Chevalier, R. A. \& Fransson, C. (1987). Nature, 328, 44-45.

Crotts, A. P. S. \& Heathcote, S. R. (1991). Nature, 350, 683-685.

Dyson, J. E. \& de Vries, J. (1972). A\&A, 20, 223-232.

Falle, S. A. E. G. (1975). A\&A, 43, 323-336.

Ginzberg, V. L. \& Syrovatskii, S. I. A. (1965). Ann. Rev. Astr. Astrophys., 3, 297-350.

Gottesman, S. T., Broderick, J. J., Brown, R. L., Balick, B. \& Palmer, P. (1972). ApJ, 174, 383-388.

Hanuschik, R. W. \& Dachs, J. (1987). A\&A, 182, L29-L30.

Jakobsen et al. (1991). ApJ, 369, L63-L66.

Jauncey, et al. (1988). Nature, 334, 412-415.

Kirk, J. G \& Wassmann, M. (1992). A\&A, 254, 167-176.

Pacholczyk, A. G. (1970). Radio Astrophysics, 171. San Francisco: Freeman.

Rupen, M. P., van Gorkom, J. H., Knapp, G. R., Gunn, J. E. \& Schneider, D. P. (1987). Astr. J., 94, 61-70.

Ryder, S., Staveley-Smith, L., Dopita, M., Petre, R., Colbert, E., Malin, D. \& Schlegel, E. (1993). ApJ, 416, 167

Staveley-Smith, L., Manchester, R. N., Kesteven, M. J., Campbell-Wilson, D., Crawford, D. F., Turtle, A. J., Reynolds, J. E., Tzioumis, A. K., Killeen, N. E. B. K. \& Jauncey, D. L. (1992). Nature, 355, 147-149.

Staveley-Smith, L., Briggs, D. S., Rowe, A. C. R., Manchester, R. N., Reynolds, J. E., Tzioumis, A. K. \& Kesteven, M. J. (1993). Nature, 366, 166.

Storey, M. C. \& Manchester, R. N. (1987). Nature, 329, 421-423.

Turtle, A. J., Campbell-Wilson, D., Bunton, J. D., Jauncey, D. L., Kesteven, M. J., Manchester, R. N., Norris, R. P., Storey, M. C. \& Reynolds, J. E. (1987). Nature, 327, 38-40.

Turtle, A. J., Campbell-Wilson, D., Manchester, R. N., Staveley-Smith, L. \& Kesteven, M. J. (1990). IAU Circular 5086.

Wang, L. \& Mazzali, P. A. (1992). Nature, 355, 58-61.

Weiler, K. W., Panagia, N., Sramek, R. A., van der Hulst, J. M., Roberts, M. S. \& Nguyen, L. (1989). ApJ, 336, 421-428.

White, G. L. \& Malin, D. (1987). ESO Workshop on SN 1987A, 11-18. Garching: ESO. 